\title{
A different view of the environment
}

\author{
Applying a method commonly used in microbiology provides a new way to study the interaction of nanoparticles \\ with environmental samples.
}

W hat is the potential hazard of nanomaterials to the environment? How many nanoparticles can be released into the environment before they start causing problems? These are basic questions, but answering them is necessary to create the appropriate regulations for the utilization of engineered - hence not naturally occurring - nanomaterials in products available to society.

Though the questions may be basic, answering them is not simple. The properties of nanoparticles, including their potential environmental effects, depend on many factors including the exact size, composition and shape. Eventually, the environmental effect of nanoparticles depends also on the specific conditions considered, and it is therefore encouraging that studies of environmental nanotechnology under realistic conditions are increasing.

One such study is included in this issue. Jacob Metch and co-workers report the results of their study of the interaction of gold nanoparticles with a microbial community extracted from wastewater sludge using metagenomic analysis, discovering that after a period of several weeks the shape of nanoparticles has a clear effect on the microbial community examined. In a nutshell, metagenomic analysis involves the high-throughput sequencing of DNA extracted from an environmental sample, and aims at capturing the diversity of a whole natural microbial community, as opposed to single types of microbial organism as typically studied using cloned cultures in the lab.

Though metagenomics is a relatively common technique in environmental microbiology, its use to study the effects of nanoparticles is a new approach and can provide information based on a large number of data that would not be possible to obtain otherwise. Metch and colleagues were especially interested in exploring the effect of metal nanoparticles coated with different substances and with different morphologies. Gold was chosen primarily due to its relative inertness, so that the effect of two types of coating and two shapes - spheres and rods - could be studied independently from the potential effects of the metal.

The particles were released in the sludge sample for a period of several weeks and

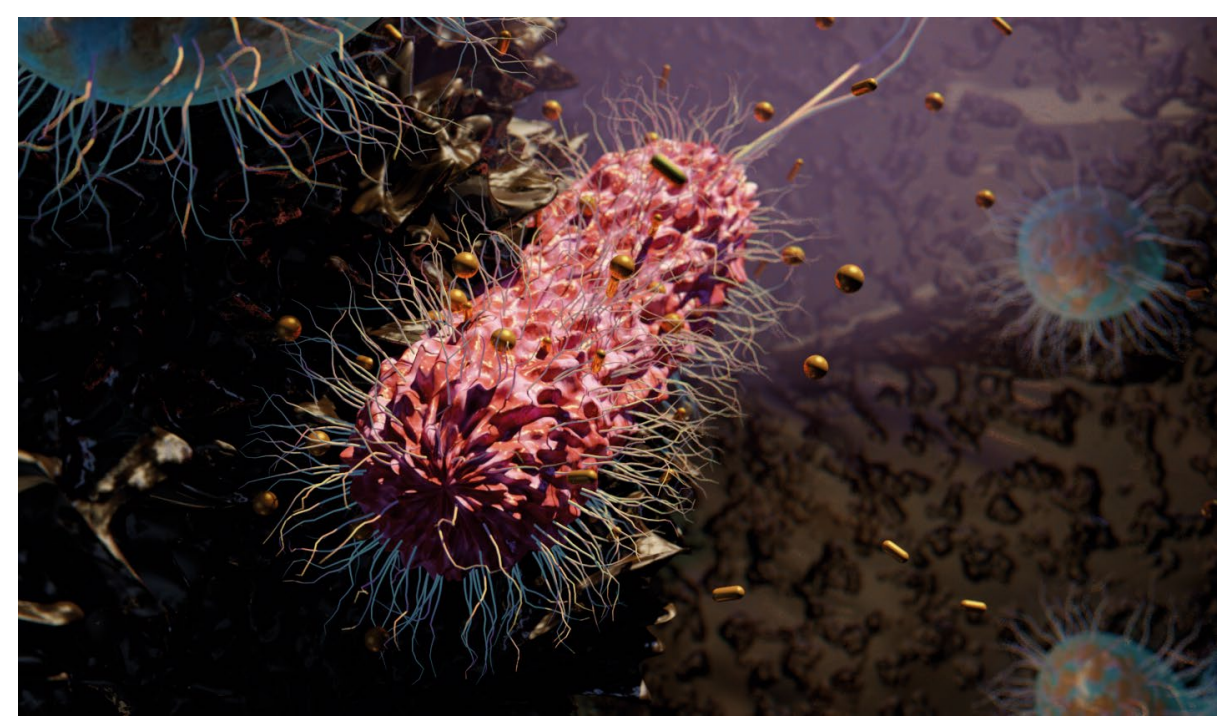

Image: Ella Maru Studio

metagenomic analysis was performed to evaluate both taxonomic and functional gene expression. The results reveal an effect of both coating and morphology, but perhaps surprisingly the effects of differences in morphology were more evident.

The work shows that by using this type of high-throughput analysis it is possible to explore general trends using, in principle, a large variety of nanomaterials. In cases like that of Metch and co-workers, the analysis can reveal unexpected effects that will be fascinating to investigate in detail. Furthermore, the fact that the analysis works by default on actual environmental samples is a particularly attractive aspect.

Perhaps indirectly, the potential effect of the nanoparticle morphology is also a corroboration of the general feeling within the environmental nanotechnology community that more specific information should be provided on nanoparticles, whether they are used in products and can be potentially released in the environment, or they are simply used in research studies reported in the scientific literature. The particle size, size distribution and shape, and eventually the methods used to determine all these specifications are but a few pieces of information that would contribute to the development of a more complete picture, improving transparency and reproducibility of results.
For scientific literature, publishers can facilitate transparency by encouraging authors to provide relevant details. Along these lines, for a few years Nature journals including Nature Nanotechnology have requested authors to fill in a checklist for life sciences experiments, detailing information such as the sample size or the number and types of replica. The document is now used as a reporting summary and is published alongside the corresponding paper. A similar document was also created for manuscripts reporting solar cells, and since 1 March 2018 a reporting summary on behavioural and social sciences has been introduced. Work is in progress on a reporting summary in ecology, evolution and environmental studies. This will be partly relevant for manuscripts in environmental nanotechnology as it will include details about the environmental sample chosen. However, at this stage the inclusion of details on the characterization of the materials involved is not being contemplated. We are fully aware that these details are more significant for publications on environmental effects of nanotechnology, and we are intending to collaborate with the scientific community to establish the best way to report essential details.

Published online: 6 March 2018 https://doi.org/10.1038/s41565-018-0106-2 